\title{
The response to teriparatide of a patient with beta-thalassemia and multiple endocrine complications
}

\author{
Cima Luminita- Nicoleta ${ }^{1,3}$, Albu Alice ${ }^{2,3}$, Lambrescu loana ${ }^{1,3}$, Barbu Carmen Gabriela ${ }^{2,3}$, Fica Simona ${ }^{2,3}$
}

${ }^{1}$ PhD student in the project Sectorial Operational Program Human Resources Development (SOPHRD), financed by the European Social Fund and the Romanian Government under the contract number POSDRU 141531;

2 Elias Hospital, Department of Endocrinology;

"Carol Davila" University of Medicine and Pharmacy.

\section{Introduction}

Osteopenia/osteoporosis in young adults with beta thalassemia major (BTM) is a prominent cause of morbidity despite adequate transfusion and iron chelation therapy. The reported frequency of osteoporosis, even in well treated TM patients varies from $13.6 \%$ to $50 \%$, with an additional $45 \%$ affected by osteopenia. There are gender differences not only in the prevalence but also in the severity of the osteoporosis, as males are more frequently and more severely affected than females.

The pathogenesis of osteoporosis in BTM is multifactorial and is related to endocrine complications (delayed puberty, hypogonadotropic hypogonadism, growth hormone and insulin-like growth factor I deficiency, hypothyroidism, parathyroid gland dysfunction, secondary diabetes), ineffective hematopoiesis with progressive marrow hyperplasia, iron toxicity on osteoblasts, direct toxic effect of desferrioxamine on bone growth, calcium, vitamin $\mathrm{D}$ and zinc deficiency, decreased physical activity and genetic factors.

Early identification and management of endocrine complications with adequate hormone replacement therapy and induction of puberty at a proper age are key points in the prevention of bone disease in patients with BTM. Because of the proven increase of bone resorption markers in BTM, bisphosphonates have been used in the management of thalassemia-induced osteoporosis. Agents that stimulate bone formation. such as teriparatide (a recombinant peptide fragment of PTH) might be useful in the management of thalassemia-induced osteoporosis, but there are few data regarding teriparatide efficiency in the literature.

Case report

November 2014

P.ML, 36 years old

The patient was submitted in our endocrine department

for follow-up after one year therapy with teriparatide for severe osteoporosis

Medical history

- at 6 months of age -diagnosed with BTM and he was treated with chronic blood transfusions since that moment;

Mean pretransfusional $\mathrm{Hb}=8.8 \mathrm{~g} / \mathrm{dl}$;

Mean post transfusional $\mathrm{Hb}=11 \mathrm{~g} / \mathrm{dl}$

- 3 ys- splenectomy

-3 ys- iron chelation therapy was initiated with desferrioxamine because the treatment was performed intermittently due to limited availability and lack of compliance of the patient the serum ferritin level was very high (6000 ng/dl);

-30 ys, therapy with deferasiroxum was started and his serum ferritin level decreased progressively from $2500 \mathrm{ng} / \mathrm{dl}$ (at initiation) to $900 \mathrm{ng} / \mathrm{dl}$ in the present time

- Liver disease related to iron overload and chronic hepatitis C

- Cardiomegaly from hemochromatosis

- Femoral dyaphysis fracture (1992)

- Hypogonadotropic hypogonadism (2008)- treated with testosterone undecanoate;

- Secondary diabetes mellitus (2008)- treated with insulin

- Hypoparathyroidism (2009)- treated with calcium and vitamin D supplements

Severe osteoporosis (2009) with multiple vertebral fractures;

$>\quad$ Vertebroplasty performed at T11 and T12;

$>\quad$ Began treatment with alendronate until Nov 2013

- Nov 2013- vertebral fracture occurred at L5;

$>\quad$ therapy with rPTH was initiated;

Physical exam

Hemosiderosic skin and jaundiced sclera;

$\mathrm{BP}=100 / 80 \mathrm{mmHg}, 76$ bpm;

Hepatomegaly,

Tanner P5G2, penis $11 \mathrm{~cm}$.

Laboratory tests

$-H \mathrm{~b}=9,2 \mathrm{~g} / \mathrm{dl}$

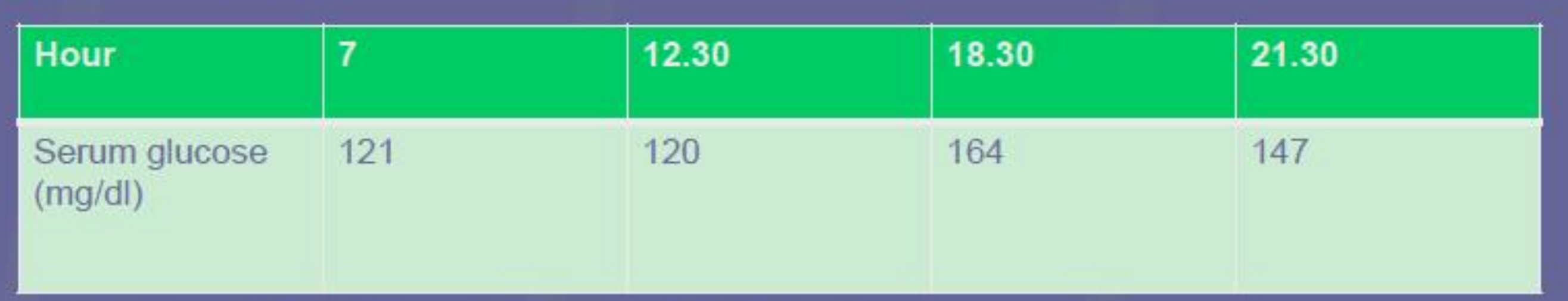

- $\mathrm{ALT}=13 \mathrm{U} / \mathrm{l} ; \quad \mathrm{AST}=15 \mathrm{U} / \mathrm{l} ;$

- Serum calcjum = $8.6 \mathrm{mg} / \mathrm{dl}(8-10.2 \mathrm{mg} / \mathrm{dl})$;

- TSH= $3.56 \mathrm{microUl} / \mathrm{ml}(0.4-4.4 \mathrm{microUl} / \mathrm{ml})$

- PTH= $13.4 \mathrm{pg} / \mathrm{ml}(19-69 \mathrm{pg} / \mathrm{ml})$;

- Testosterone $=758 \mathrm{ng} / \mathrm{dl}($ 300-1000 ng/dl) under chronic substitutive treatment.

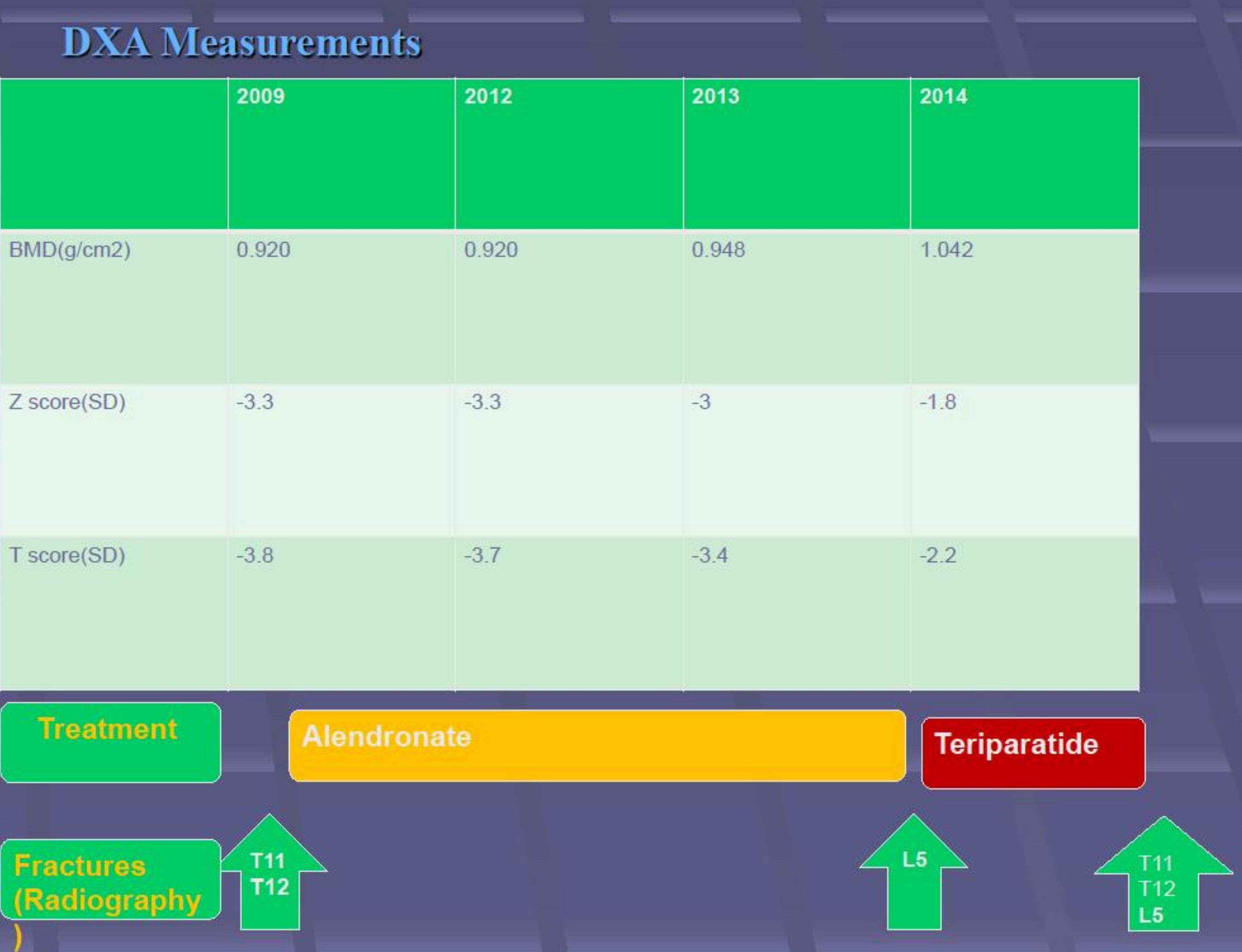

In spite the fact our patient received substitutive therapy with testosterone for hypogonadism and calcium and vitamin $\mathrm{D}$ for hypoparathyroidism and the diabetes was treated with insulin with appropriate doses he presented a severe bone disease at a young age (31ys) with 2 vertebral fractures (T11, T12).

During treatment with bisphosphonates, a new vertebral fracture occurred (L5); therefore, therapy with $\mathrm{PPTH}$ was initiated.

DXA measured lumbar spine BMD significantly improved ( $9.8 \%$ increment) after 1 year of treatment with teriparatide; the lumbar spine radiography showed no signs of a new fracture.

Conctusions

Despite improvement of treatment, the sequelae of osteoporosis are a major cause of morbidity in BTM patients. A better understanding of the pathogenic mechanisms are of paramount importance for the management of these patients

The osteoporosis in BTM has a complex mechanism; both increased bone resorption and decreased formation being involved. Probably the extent of contribution of the two mechanisms varies in each patient.

Although the bisphosphonates are the most used treatment for osteoporosis in BTM, our case report suggests that, at least in some patients, decreased bone formation is the predominant mechanism and teriparatide could be the most appropriate therapy.

ACKNOWLEDGEMJENTJ: This paper is partly supported by the Sectorial Operational Programme Human Resources Development (SOPHRD), financed by the European Social Fund and the Romanian Government under the contract number POSDRU 141531.

References

De Sanctis, V., et al., Osteoporosis in thalassemia major: an update and the ICET 2013 recommendations for surveillance and treatment. Pediatr Endocrino Rev, 2013, 11(2): p. 167-80

Perisano, C., et al., Physiopathology of Bone Modifications in betaThalassemia. Anemia, 2012. 2012: p. 320737.

Baldini. M. et al., Endocrine and bone disease in appropriately treated adult patients with beta-thalassemia major. Ann Hematol, 2010. 89(12): p. 1207-13

Voskaridou, E. and E. Terpos, Pathogenesis and management of osteoporosis in thalassemia. Pediatr Endocrinol Rev, 2008. 6 Suppl 1: p. 86-93

Skordis, N. and M. Toumba, Bone disease in thalassaemia major: recent advances in pathogenesis and clinical aspects. Pediatr Endocrinol Rev, 2011. \& Suppl 2: p. 300-6. 